\title{
Phonons in aluminum at high temperatures studied by inelastic neutron scattering
}

\author{
M. Kresch, M. Lucas, O. Delaire, J. Y. Y. Lin, and B. Fultz \\ W. M. Keck Laboratory, California Institute of Technology, Mail 138-78, Pasadena, California 91125, USA
}

(Received 14 August 2007; published 3 January 2008)

\begin{abstract}
Inelastic neutron scattering measurements on aluminum metal were performed at temperatures of 10,150 , 300, 525, and $775 \mathrm{~K}$ using direct-geometry Fermi chopper spectrometers. The temperature dependent phonon density of states (DOS) was determined from the scattering, and was used to fit Born-von Kármán models of lattice dynamics. The shifts in the phonon frequencies with increasing temperature were largely explained by the softening of the longitudinal force constants out to third nearest neighbors. A significant broadening of the phonon spectra at high temperatures was also measured. The phonon DOS was used to determine the vibrational contributions to the entropy of aluminum as a function of temperature. All other contributions to the entropy of aluminum were calculated or assessed, and the total entropy was in excellent agreement with the NIST-JANAF compilation [M. W. Chase, J. Phys. Chem. Ref. Data Monogr. 9, 59 (1998)]. Anharmonic effects were attributed to phonon-phonon interactions. The quasiharmonic approximation was generally successful, but its weaknesses are discussed.
\end{abstract}

DOI: 10.1103/PhysRevB.77.024301

PACS number(s): 63.20.D-, 61.05.fg, 65.40.G-

\section{INTRODUCTION}

Due to its abundance and the favorable thermal and mechanical properties of its alloys, aluminum is one of the most widely used metals for industrial and engineering applications. Its melting temperature of $933 \mathrm{~K}$ is relatively low, and the thermodynamic stability of aluminum at elevated temperatures is of technological and scientific importance. Because of its simple electronic structure, aluminum metal is frequently used as a test case for theoretical models of crystals and their thermodynamics. ${ }^{1-10}$

Aluminum is nonmagnetic, so the majority of its entropy comes from phonons. In turn, the majority of its phonon entropy $S_{\mathrm{ph}}$ can be attributed to harmonic oscillations of the nuclei about their equilibrium positions. The "quasiharmonic" phonon entropy $S_{\mathrm{ph}, \mathrm{Q}}$ includes both the harmonic phonon entropy and the entropy due to a decrease in phonon frequencies (softening) as the crystal expands. Measurements of phonon dispersions in body centered cubic metals ${ }^{11-16}$ have shown that the quasiharmonic model is often insufficient to explain the temperature dependence of the phonon entropy. For example, $S_{\mathrm{ph}, \mathrm{Q}}$ is an overestimate of the phonon entropy in both niobium ${ }^{17}$ and vanadium, ${ }^{12}$ but a severe underestimate for chromium. ${ }^{17}$ There is less experimental data on the high temperature trends in phonons and phonon entropy in face-centered cubic (fcc) metals, although recent work has shown that the phonons in nickel are slightly stiffer than predicted by the expansion of the lattice against the bulk modulus. ${ }^{18}$

Aluminum has one natural isotope, ${ }^{27} \mathrm{Al}$, and scatters thermal neutrons coherently. There have been a number of measurements of its phonon dispersions using neutron triple-axis spectrometers, ${ }^{19-23}$ and other work using x-ray diffuse scattering. ${ }^{24,25}$ The phonon dispersions at 80 and $300 \mathrm{~K}$ measured with inelastic neutron scattering by Stedman and Nilsson ${ }^{23}$ have been used frequently, sometimes to generate the phonon density of states (DOS). ${ }^{2,5,26-28}$

Previous measurements of phonons in aluminum at temperatures above $300 \mathrm{~K}$ were limited to small numbers of momentum transfers. Because aluminum is a coherent scatterer, such measurements sampled only small numbers of phonon states, and are not optimal for determining the phonon DOS that is so important for thermodynamics. Nevertheless, Larsson et al. ${ }^{22}$ found that some phonon frequencies shifted by approximately $15 \%$ between temperatures of 298 and $932 \mathrm{~K}$. They found phonon linewidths to increase at temperatures above $600 \mathrm{~K}$. Peterson and $\mathrm{Smith}^{25}$ also suggested that the longitudinal modes are anharmonic.

Energy shifts and lifetime broadening of phonons in aluminum and other fcc metals have been studied theoretically. ${ }^{29-34}$ Björkman et al., using a pseudopotential model, ${ }^{31}$ show how electron-phonon interactions shorten phonon lifetimes in aluminum. Using Born-von Kármán $(\mathrm{BvK})$ fits to neutron data in conjunction with measurements of second and third order elastic constants, Zoli et al. calculated the shifts ${ }^{32}$ and lifetime broadening ${ }^{33}$ of the phonons in aluminum at temperatures below room temperature. More generally, understanding the contributions of quasiparticles and collective excitations to the free energy, entropy, and heat capacity of crystalline solids is an active area of research. ${ }^{17,35-44}$

Here we present results from inelastic neutron scattering measurements of the phonon DOS of aluminum at temperatures of $10,150,300,525$, and $775 \mathrm{~K}$. We use these results to determine the phonon contributions to the entropy of aluminum, and we assess the other entropic contributions, finally obtaining excellent agreement with the total thermodynamic entropy. The overall softening of the phonons is found to be caused by a monotonic temperature dependence of the first nearest-neighbor $(1 \mathrm{NN})$, second nearest-neighbor $(2 \mathrm{NN})$, and third nearest-neighbor $(3 \mathrm{NN})$ force constants, with the $1 \mathrm{NN}$ force constants decreasing approximately $10 \%$ over the temperature range of measurement. The purely anharmonic part of the phonon entropy, not accounted for by the expansion of the lattice, is approximately $-0.07 k_{B} /$ atom at $775 \mathrm{~K}$. Additionally, we quantify the temperature dependence of the energy widths and shifts of the phonons, and we attribute the anharmonic effects to phonon-phonon interactions. 


\section{COMPONENTS OF THE ENTROPY}

As temperature increases, the entropic terms in the free energies of materials become increasingly important. At the same time, accurate assessment of the different components of the entropy becomes increasingly complicated. It is frequently assumed that the states of the system may be split into combinations of independent subsystems of states such that

$$
S=k_{B} \ln \Omega \approx k_{B} \ln \left(\prod_{i} \Omega_{i}\right)=k_{B} \sum_{i} \ln \left(\Omega_{i}\right)=\sum_{i} S_{i},
$$

where $\Omega$ is the number of states of the system, $\Omega_{i}$ is the number of states of the subsystem $i$, and $S_{i}$ is the entropy of subsystem $i$. Especially at high temperatures, it may be necessary to correct for the interactions between the subsystems. (These corrections represent differences between simplified models and reality, and may thus be positive or negative. They are called "entropy" nevertheless.)

For a nonmagnetic crystalline solid, the total entropy $S$ may be broken up into contributions from electrons $S_{\mathrm{el}}$, phonons $S_{\mathrm{ph}}$, and their interactions $S_{\mathrm{el}-\mathrm{ph}}$, with the phonon entropy usually dominant at high temperatures. For a polyatomic solid, the configurational entropy $S_{c}$ is also significant, but for crystals of a pure element, the configurational contribution arises from defects. In either case, we have

$$
S=S_{\mathrm{ph}}+S_{\mathrm{el}}+S_{\mathrm{el}-\mathrm{ph}}+S_{c} .
$$

The quasiharmonic entropy includes both the harmonic and dilatational contributions to the phonon entropy, $S_{\mathrm{ph}, \mathrm{H}}$ and $S_{\mathrm{ph}, \mathrm{D}}$, respectively. Any additional changes of the phonon entropy with increasing temperature are termed the anharmonic entropy, $S_{\mathrm{ph}, A}$, so

$$
S_{\mathrm{ph}}=S_{\mathrm{ph}, \mathrm{H}}+S_{\mathrm{ph}, \mathrm{D}}+S_{\mathrm{ph}, A}=S_{\mathrm{ph}, \mathrm{Q}}+S_{\mathrm{ph}, A} .
$$

It is sometimes useful to consider the nonharmonic phonon entropy $S_{\mathrm{ph}, \mathrm{NH}}$, which is the sum of the dilatation and anharmonic contributions

$$
S_{\mathrm{ph}, \mathrm{NH}}=S_{\mathrm{ph}, \mathrm{D}}+S_{\mathrm{ph}, A}=S_{\mathrm{ph}}-S_{\mathrm{ph}, \mathrm{H}} .
$$

Similar divisions may be made for the electronic entropy,

$$
S_{\mathrm{el}}=S_{\mathrm{el}, \mathrm{G}}+S_{\mathrm{el}, \mathrm{D}}
$$

where $S_{\mathrm{el}, \mathrm{G}}$ is the ground state electron entropy and $S_{\mathrm{el}, \mathrm{D}}$ is the electronic entropy associated with changes in the electron DOS from dilatation of the lattice. Both of these contributions to the electronic entropy originate with noninteracting electrons.

The electron-phonon entropy is separated into two parts:

$$
S_{\text {el-ph }}=S_{\text {el-ph,na }}+S_{\text {el-ph,ad }} \text {. }
$$

The nonadiabatic electron-phonon entropy $S_{\text {el-ph,na, which }}$ dominates at low temperatures, is associated with the mixing of the electron ground states from the nuclear motion. At higher temperatures, the adiabatic electron-phonon entropy $S_{\text {el-ph,ad }}$ dominates. It accounts for the thermal shifts of electron states caused by average nuclear motions. These contributions to the entropy have been discussed in greater detail previously. ${ }^{40-46}$ There should also be a contribution to the electron-phonon entropy from the dilatation of the lattice; however, we expect this to be negligible.

For pure, polycrystalline, metallic aluminum, we expect the configurational entropy $S_{c}$ to be nearly equal to the configurational entropy of vacancies $S_{\mathrm{vac}, c}$. Putting this all together,

$$
\begin{aligned}
S \approx & S_{\mathrm{ph}, \mathrm{H}}+S_{\mathrm{ph}, \mathrm{D}}+S_{\mathrm{ph}, A}+S_{\mathrm{el}, \mathrm{G}} \\
& +S_{\mathrm{el}, \mathrm{D}}+S_{\mathrm{el-ph,ad}}+S_{\mathrm{el-ph,na}}+S_{\mathrm{vac}, c} .
\end{aligned}
$$

\section{EXPERIMENT, ANALYSIS, AND COMPUTATION}

Clean aluminum shot of $99.99 \%$ purity was arranged to cover maximally the interior of a thin-walled, rectangular, aluminum pan, whose height, width, and depth were approximately $10.0,7.0$, and $0.5 \mathrm{~cm}$. The ratio of singly to multiply scattered neutrons was designed to be approximately $10 \%$.

One set of inelastic neutron scattering measurements was performed with the Low Resolution Medium Energy Chopper Spectrometer (LRMECS) at the Intense Pulsed Neutron Source at Argonne National Laboratory. The aluminum pan was mounted at $45^{\circ}$ with respect to the incident neutron beam, and measurements were made at 10, 150, 300, 525, and $775 \mathrm{~K}$. Measurements were also made on the empty aluminum pan at all temperatures to allow for some removal of background scattering. All measurements were approximately $8 \mathrm{~h}$ in duration, and included approximately 750000 counts. For the lower temperatures, 10, 150, and $300 \mathrm{~K}$, the sample was mounted in a displex refrigerator. For the higher temperatures, the sample was mounted in a low background, electrical resistance furnace designed for vacuum applications. In both cases, temperature was monitored with several thermocouples, and is believed accurate to within $5 \mathrm{~K}$ over the bulk of the sample. The incident neutron energy, determined from the scattering, was $59.1 \mathrm{meV}$.

A second set of measurements was made with the Pharos time-of-flight chopper spectrometer at the Los Alamos Neutron Science Center. Another displex refrigerator was used for a measurement at $10 \mathrm{~K}$, and the same furnace was used for a measurement at $775 \mathrm{~K}$. The same aluminum sample was used, again mounted at an angle of $45^{\circ}$ with respect to the incident neutron beam. Data were collected for a minimum of $4 \mathrm{~h}$ at each temperature, giving on the order of 1 $\times 10^{6}$ counts. The incident neutron energy was $69.3 \mathrm{meV}$. For both instruments, the energy resolution function $R\left(E, E^{\prime}\right)$ was assumed to be Gaussian in energy,

$$
R\left(E, E^{\prime}\right)=\frac{1}{\sigma_{I}\left(E^{\prime}\right) \sqrt{2 \pi}} \exp \left\{-\frac{1}{2}\left[\frac{E-E^{\prime}}{\sigma_{I}\left(E^{\prime}\right)}\right]^{2}\right\} .
$$

The widths were approximated from the timing characteristics of the two instruments as

$$
\begin{gathered}
\sigma_{\text {LRMECS }}\left(E^{\prime}\right) \approx-0.0071 E^{\prime}+1.2744, \\
\sigma_{\text {Pharos }}\left(E^{\prime}\right) \approx-0.0091 E^{\prime}+1.0620,
\end{gathered}
$$

where $E^{\prime}$ and $\sigma\left(E^{\prime}\right)$ are in $\mathrm{meV}$.

The raw data from both instruments, in time-of-flight and scattering angle $2 \theta$ were first normalized using the counts in 
TABLE I. Experimentally determined lattice parameter, $a$, of aluminum and shifts of the aluminum phonon energies as a function of temperature. Fits of the $10 \mathrm{~K}$ DOS to the high temperature DOS by scaling of the energy and convolution (Ref. 49) with the damped oscillator function yield the relative frequency shifts $\lambda$ [Eq. (13)]. $\left\langle E_{T}\right\rangle /\left\langle E_{10}\right\rangle$ are ratios of the first moments of the DOS [Eq. (15)]. Values in square brackets are from Pharos data.

\begin{tabular}{lccccc}
\hline \hline $\begin{array}{l}T \\
(\mathrm{~K})\end{array}$ & $\begin{array}{c}a \pm 0.01 \\
(\AA)\end{array}$ & $\lambda$ & $\left\langle E_{T}\right\rangle /\left\langle E_{10}\right\rangle$ & $S_{\mathrm{ph}} \pm 0.03$ & $\left(k_{B} /\right.$ atom $)$ \\
\hline 10 & 4.041 & 1.000 & 1.000 & 0.001 & {$[0.001]$} \\
150 & 4.045 & 0.995 & 0.990 & 1.628 & \\
300 & 4.056 & 0.977 & 0.964 & 3.462 & \\
525 & 4.079 & 0.954 & 0.941 & 5.146 & {$[6.306]$} \\
775 & 4.111 & 0.952 & 0.941 & 6.332 & \\
\hline \hline
\end{tabular}

the beam monitor or the integrated proton current. Bad detectors were identified and masked, and the data were corrected for detector efficiency using a measurement of vanadium, an incoherent scatterer. At each temperature, the measured scattering from the empty aluminum pan was subtracted from the data, reduced by roughly $10 \%$ to account for the self-shielding of the sample. The data were then binned to get intensity $I(E, 2 \theta)$ as a function of scattering angle $2 \theta$ and energy $E$ transferred to the sample. Approximately, $2 \theta$ ranged from $10^{\circ}$ to $120^{\circ}$ with a bin width of $0.75^{\circ}$, and $E$ ranged from -60.0 to $60.0 \mathrm{meV}$ with a bin width of $0.5 \mathrm{meV}$ for both instruments.

By summing the LRMECS data from -5.0 to $5.0 \mathrm{meV}$, in situ neutron diffraction patterns were obtained. Lattice parameters were determined from these data using Nelson-Riley ${ }^{47}$ plots, and are listed in Table I. Their thermal trends are consistent with thermal expansion data.

Data reduced to $I(E, 2 \theta)$ were then rebinned into intensity, $I(Q, E)$, where $Q$ is the momentum transferred to the sample. For both instruments, $Q$ ranged from 0.0 to $12 \AA^{-1}$, with a bin width of about $0.075 \AA^{-1}$. The elastic peak was removed below $8 \mathrm{meV}$ and replaced by a function $\xi(E)$ of the form

$$
\xi(E) \approx \frac{A E}{1-\exp (-\beta E)},
$$

where the constant $A$ was determined from the inelastic scattering just past the elastic peak. Here, we have assumed that

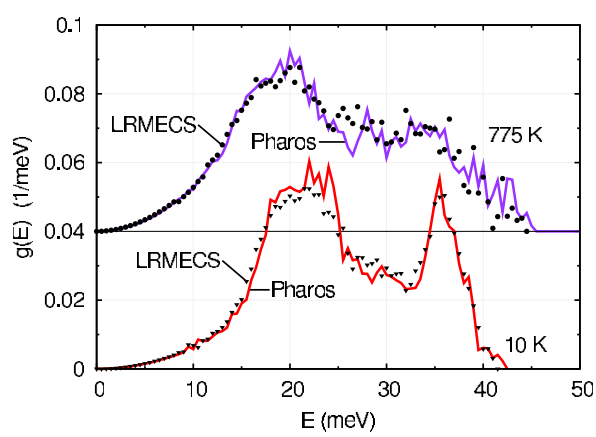

FIG. 1. (Color online) Phonon DOS of aluminum at temperatures as indicated. The markers show the experimentally determined DOS from LRMECS, and the lines show the DOS from Pharos. the phonon DOS is proportional to $E^{2}$ in the low energy regime, as in a Debye model. The phonon DOSs were then extracted from the scattering, making the thermal corrections and corrections for multiphonon and multiple scattering, as described previously. ${ }^{18}$ These phonon DOSs are shown by markers and lines in Fig. 1, and by the markers in Fig. 2.

With increasing temperature, the phonon peaks in a metallic solid typically broaden and undergo a shift to lower energies. These shifts were approximated as a constant multiplier $\lambda$ applied to all phonon energies $E$ :

$$
E \rightarrow \lambda E
$$

The broadening of the phonons was assumed to take the form of a damped harmonic oscillator function $D\left(\mathcal{Q}, E^{\prime}, E\right)$ centered about energy ${ }^{48} E^{\prime}$ :

$$
D\left(\mathcal{Q}, E^{\prime}, E\right)=\frac{1}{\pi \mathcal{Q} E^{\prime}} \frac{1}{\left(\frac{E^{\prime}}{E}-\frac{E}{E^{\prime}}\right)^{2}+\frac{1}{\mathcal{Q}^{2}}} .
$$

Using Eqs. (11) and (12), the high temperature phonon DOS was approximated as a function of the low temperature DOS, with only two free parameters, $\lambda$ and $\mathcal{Q}$ :

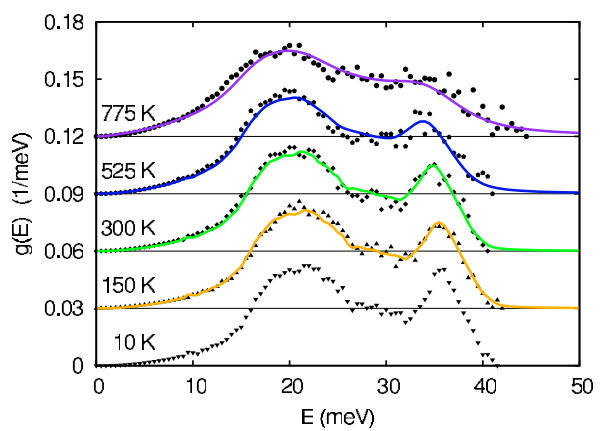

FIG. 2. (Color online) Phonon DOS of aluminum at temperatures as indicated. The markers show the experimentally determined DOS, and the lines the best fits of the $10 \mathrm{~K}$ DOS to the high temperature DOS by scaling of the energy and convolution (Ref. 49) with the damped oscillator function [Eq. (13)]. 


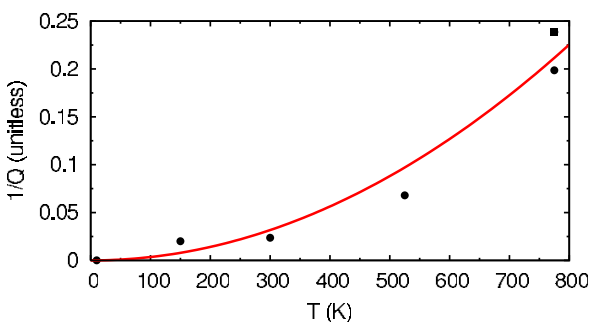

FIG. 3. (Color online) Markers show the inverse of the quality factor, $1 / \mathcal{Q}$, as a function of temperature for aluminum phonons, circles are LRMECS data, and the square is Pharos data. The line is a parabolic fit [Eq. (14)].

$$
g_{T}(E)=D\left(\mathcal{Q}, E^{\prime}, E\right) * g_{10}\left(\lambda E^{\prime}\right),
$$

where $g_{T}$ is the phonon DOS at temperature $T$, and $*$ denotes an integral transform that is similar to a convolution. ${ }^{49}$ (The subscript 10 , as in $10 \mathrm{~K}$, refers to the lowest temperature data from this set of experiments.)

At each temperature, the best $\mathcal{Q}$ and $\lambda$ for the experimental DOS were determined through a least squares algorithm. The $\mathcal{Q}$ so determined are shown in Fig. 3, and the fits to the phonon DOS are shown in Fig. 2. The inverse of the quality factor was well described by a quadratic function of $T$ :

$$
\frac{1}{\mathcal{Q}} \approx 3.523 \times 10^{-7} T^{2},
$$

where $T$ is in degrees Kelvin. The shifts $\lambda$ are given in Table I, along with the ratios of the mean phonon energies, $\left\langle E_{T}\right\rangle /\left\langle E_{10}\right\rangle$, as determined from the DOS:

$$
\frac{\left\langle E_{T}\right\rangle}{\left\langle E_{10}\right\rangle}=\frac{\int E g_{T}(E) d E}{\int E g_{10}(E) d E} .
$$

In a second analysis, the phonon DOSs were fitted with Born-von Kármán models of the lattice dynamics. ${ }^{50-53}$ Tensorial force constants to the $3 \mathrm{NN}$ shell were determined with a gradient search method. For the higher temperatures, where there is significant anharmonic broadening, these models

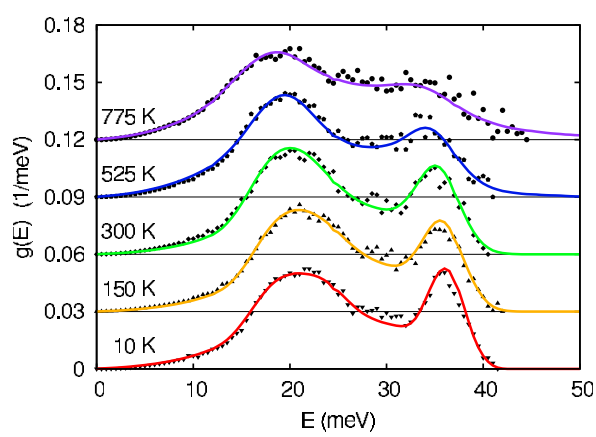

FIG. 4. (Color online) Phonon DOS of aluminum at temperatures as indicated. The markers show the experimentally determined DOS, and the lines the best BvK models found by fitting the data.

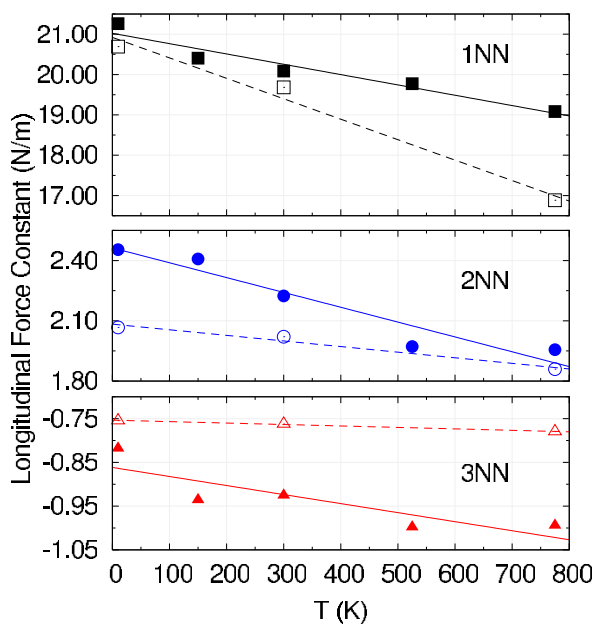

FIG. 5. (Color online) Longitudinal force constants for fcc aluminum as a function of temperature. Filled markers and solid lines are from BvK fits, unfilled markers and dashed lines are from calculations using VASP (Refs. 54 and 55) and PHON (Ref. 58). The fits to the BvK force constants are given by Eqs. (16)-(18). For the BvK models, the higher order force constants were fixed as described in the text.

were sufficiently accurate. At lower temperatures, however, they were unable to reproduce the shape of the DOS. For the DOS at 10,150 , and $300 \mathrm{~K}$, axially symmetric force constants from fourth to eight nearest-neighbor shells were also optimized. These showed little change with temperature, so they were averaged and kept constant for a final round of optimization.

To account for the thermal and instrument broadening, the frequencies $\omega$, calculated as the square root of each eigenvalue of the dynamical matrix, were taken to be the arguments of delta functions in energy, $\delta(E-\hbar \omega)$. Each delta function was convolved ${ }^{49}$ with the damped harmonic oscillator function of Eq. (12), and the Gaussian instrument resolution function given by Eqs. (8) and (9). The force constants so determined are listed in Table II, and the best fits to the DOS at all temperatures are shown in Fig. 4.

The longitudinal force constants were found by projecting the tensor onto the bond vectors $\langle x y z\rangle$. The $3 \times 3$ force constant tensors were then diagonalized. The longitudinal force constant was matched to one of the eigenvalues, and the

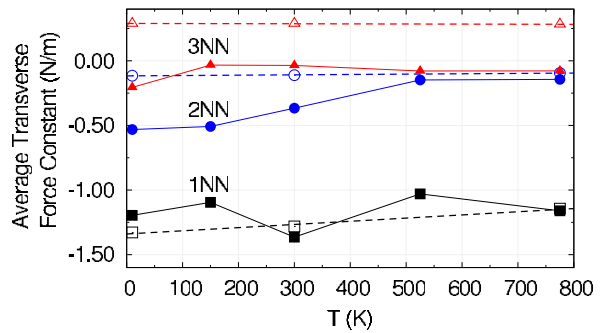

FIG. 6. (Color online) Averaged transverse force constants for fcc aluminum as a function of temperature. Filled markers and solid lines are from BvK fits, unfilled markers and dashed lines are from calculations using VASP (Refs. 54 and 55) and PHON (Ref. 58). 
TABLE II. Optimized tensor force constants in N/m as a function of temperature for fcc aluminum. They are given in a Cartesian basis, where $\langle x y z\rangle$ is the bond vector for the given tensor components.

\begin{tabular}{|c|c|c|c|c|c|c|}
\hline & $\langle x y z\rangle$ & $10 \mathrm{~K}$ & $150 \mathrm{~K}$ & $300 \mathrm{~K}$ & $525 \mathrm{~K}$ & $775 \mathrm{~K}$ \\
\hline$\Phi_{1 x x}$ & $\langle 110\rangle$ & 10.112 & 9.708 & 9.708 & 10.542 & 10.112 \\
\hline$\Phi_{1 x y}$ & & 11.148 & 10.697 & 10.378 & 9.232 & 8.970 \\
\hline$\Phi_{1 z z}$ & & -1.356 & -1.201 & -2.059 & -3.370 & -3.463 \\
\hline$\Phi_{2 x x}$ & $\langle 200\rangle$ & 2.454 & 2.408 & 2.224 & 1.972 & 1.956 \\
\hline$\Phi_{2 y y}$ & & -0.532 & -0.508 & -0.367 & -0.148 & -0.144 \\
\hline$\Phi_{3 x x}$ & $\langle 211\rangle$ & -0.634 & -0.636 & -0.635 & -0.707 & -0.706 \\
\hline$\Phi_{3 x y}$ & & -0.185 & -0.301 & -0.294 & -0.301 & -0.299 \\
\hline$\Phi_{3 y y}$ & & -0.298 & -0.183 & -0.181 & -0.225 & -0.222 \\
\hline$\Phi_{3 y z}$ & & -0.149 & -0.147 & -0.148 & -0.151 & -0.151 \\
\hline$\Phi_{4 x x}$ & $\langle 220\rangle$ & 0.273 & 0.273 & 0.273 & 0.000 & 0.000 \\
\hline$\Phi_{4 x y}$ & & -0.051 & -0.051 & -0.051 & 0.000 & 0.000 \\
\hline$\Phi_{4 z z}$ & & 0.324 & 0.324 & 0.324 & 0.000 & 0.000 \\
\hline$\Phi_{5 x x}$ & $\langle 310\rangle$ & 0.469 & 0.469 & 0.469 & 0.000 & 0.000 \\
\hline$\Phi_{5 x y}$ & & 0.090 & 0.090 & 0.090 & 0.000 & 0.000 \\
\hline$\Phi_{5 y y}$ & & 0.229 & 0.229 & 0.229 & 0.000 & 0.000 \\
\hline$\Phi_{5 z z}$ & & 0.199 & 0.199 & 0.199 & 0.000 & 0.000 \\
\hline$\Phi_{6 x x}$ & $\langle 222\rangle$ & 0.144 & 0.144 & 0.144 & 0.000 & 0.000 \\
\hline$\Phi_{6 x y}$ & & -0.110 & -0.110 & -0.110 & 0.000 & 0.000 \\
\hline$\Phi_{7 x x}$ & $\langle 321\rangle$ & -0.061 & -0.061 & -0.061 & 0.000 & 0.000 \\
\hline$\Phi_{7 x y}$ & & 0.032 & 0.032 & 0.032 & 0.000 & 0.000 \\
\hline$\Phi_{7 x z}$ & & 0.016 & 0.016 & 0.016 & 0.000 & 0.000 \\
\hline$\Phi_{7 y y}$ & & -0.088 & -0.088 & -0.088 & 0.000 & 0.000 \\
\hline$\Phi_{7 y z}$ & & 0.011 & 0.011 & 0.011 & 0.000 & 0.000 \\
\hline$\Phi_{7 z z}$ & & -0.105 & -0.105 & -0.105 & 0.000 & 0.000 \\
\hline$\Phi_{8 x x}$ & $\langle 400\rangle$ & -0.536 & -0.536 & -0.536 & 0.000 & 0.000 \\
\hline$\Phi_{8 v v}$ & & -0.117 & -0.117 & -0.117 & 0.000 & 0.000 \\
\hline
\end{tabular}

average transverse constant was taken to be the mean of the remaining eigenvalues. Longitudinal force constants to $3 \mathrm{NN}$ are shown in Fig. 5, and the averaged transverse force constants out to $3 \mathrm{NN}$ are shown in Fig. 6. The $X \mathrm{NN}$ longitudinal force constants, $\mathcal{L}_{X}(T)$, decrease with increasing temperature approximately as

$$
\begin{gathered}
\mathcal{L}_{1}(T)=21.022-2.559 \times 10^{-3} T, \\
\mathcal{L}_{2}(T)=2.463-7.384 \times 10^{-4} T, \\
\mathcal{L}_{3}(T)=-0.862-2.066 \times 10^{-4} T,
\end{gathered}
$$

where $T$ is in degrees Kelvin and $\mathcal{L}_{X}(T)$ is in $\mathrm{N} / \mathrm{m}$. The fits are also shown in Fig. 5.

In a third computational effort, we used the plane-wave code VASP (Refs. 54 and 55) to calculate the electronic DOS of aluminum as a function of unit cell volume. The calculations used projector augmented plane waves and the PerdewBurke-Ernzerhof generalized gradient approximation. ${ }^{56} \mathrm{~A}$ conventional fcc cell was used, and it was relaxed using the "accurate" setting for the kinetic energy cutoff, with a 20 $\times 20 \times 20$ Monckhorst-Pack $k$-point grid. ${ }^{57}$ The relaxed volume (which matched the experimentally determined volume to better than $0.2 \%$ ) was taken to be the $0 \mathrm{~K}$ volume of the unit cell, and the values of the linear coefficient of thermal expansion from Wang and Reeber ${ }^{1}$ were used to determine the volumes at temperatures corresponding to our experiments. At each of these volumes, the electronic DOS was determined on a larger, $70 \times 70 \times 70 k$-point grid. The electronic DOS at 10 and $775 \mathrm{~K}$ are shown in Fig. 7.

The interatomic forces and the phonons were calculated from first principles in the direct method using VASP in conjunction with the program PHON. ${ }^{58}$ At volumes consistent with the temperatures 0,300 , and $775 \mathrm{~K}$, the total energy was minimized for a $4 \times 4 \times 4$ (64 atom) supercell with a 4 $\times 4 \times 4$ electronic $k$-point grid. A single displacement whose length was $1 \%$ of the interatomic separation was used, and testing showed that the effects of the size of the displacement on the calculated force constants was negligible. The longi- 


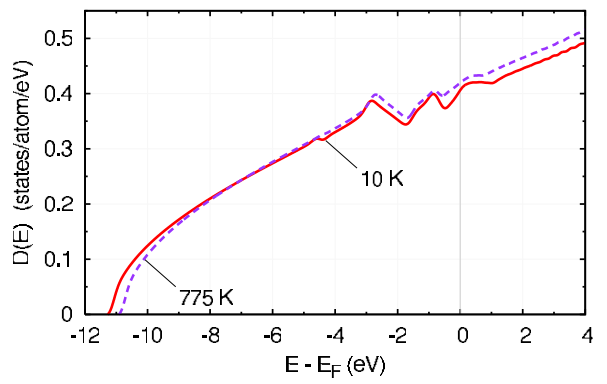

FIG. 7. (Color online) Electronic DOS of fcc aluminum at 10 and $775 \mathrm{~K}$, showing the effects of the expansion of the lattice with increasing temperature. $E_{F}$ is the Fermi energy.

tudinal and average transverse force constants were determined as for the BvK models, and are shown in Figs. 5 and 6 , respectively. The values of the longitudinal force constants to $3 \mathrm{NN}$ at $0 \mathrm{~K}$ are given in Table III.

\section{RESULTS}

The force constants obtained from BvK models need not be unique, especially for more distant nearest-neighbor shells and for off-bond directions. ${ }^{26}$ Nevertheless, Wallis et al. found that their BvK models for aluminum showed reasonable agreement with pseudopotential calculations for longitudinal force constants of the first three nearest-neighbor shells. ${ }^{26}$ Table III presents the longitudinal force constants from the pseudopotential and empirical models (at 0 and $80 \mathrm{~K}$, respectively) of Wallis et al., from the empirical model (at $80 \mathrm{~K}$ ) of Gilat and Nicklow, from our empirical model (at $10 \mathrm{~K}$ ), and from our plane-wave calculations (at $0 \mathrm{~K}$ ). The table shows remarkable agreement of the major longitudinal force constants, giving confidence in our values of these force constants as a function of temperature.

As seen in Fig. 5, thermal changes in the 1NN force constants are dominant, and are expected to have the largest effect in shifting the DOS to lower energies with increasing temperature. The transverse force constants shown in Fig. 6 have small changes with temperature. They have negative signs, indicating some instability in the off-bond directions. At all temperatures, the magnitudes of both the longitudinal and transverse force constants decrease rapidly with increasing distance in the first two or three shells. The behavior of the fit force constants for longer bonds is less structured, owing mainly to noise in the data and the difficulty in fitting parameters that have smaller impacts on the DOS. Nevertheless, fluctuations of sign could be consistent with Friedel oscillations in $\mathrm{Al}^{26}$

The frequencies of the transverse modes and longitudinal modes have slightly different temperature dependencies, leading to modest differences in the second and third columns of Table I. Stedman et al. ${ }^{27}$ reported $\left\langle E_{300}\right\rangle /\left\langle E_{80}\right\rangle$ $=0.98$, which seems in reasonable agreement with the value of 0.969 , obtained as the average of our values at 10 and $150 \mathrm{~K}$. A previous study by Larsson et al. ${ }^{22}$ found significantly larger shifts in the mean frequency at higher temperatures: $\left\langle E_{775}\right\rangle /\left\langle E_{300}\right\rangle=0.925,{ }^{59}$ where we find $\left\langle E_{775}\right\rangle /\left\langle E_{300}\right\rangle$

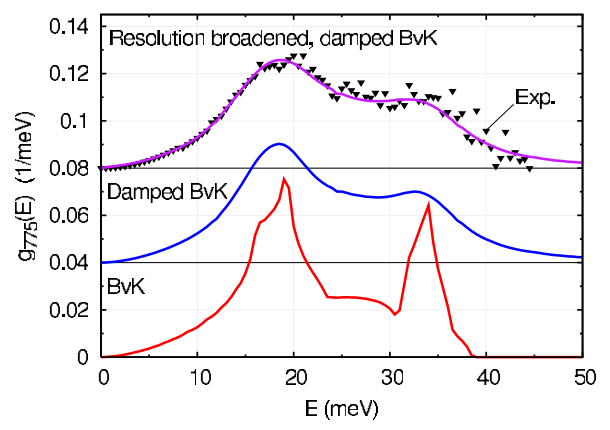

FIG. 8. (Color online) Phonon DOS for aluminum at $775 \mathrm{~K}$. Markers show the experimental data. Lines show BvK models without damping, with damping, and with both damping and instrument resolution broadening, for the bottom, middle, and top curves, respectively.

$=0.976$, perhaps because these early results were based on the central energies of broadened phonon peaks, and also because the much greater region of $Q$ space sampled in the present measurements gives a better average of the phonon softening.

From the values of $1 / \mathcal{Q}$ shown in Fig. 3, at the highest energy of the phonons, $38.0 \mathrm{meV}$, we find maximum values of the full width at half maximum $2 \Gamma=E / \mathcal{Q}$ to be approximately $0,0.8,0.9,2.6$, and $7.5 \mathrm{meV}$ at the temperatures 10 , $150,300,525$, and $775 \mathrm{~K}$, respectively. This broadening seems consistent with the experimental values reported by Larsson et al. ${ }^{22}$ Linewidths due to phonon-phonon interactions for aluminum at $300 \mathrm{~K}$ were calculated by Zoli et al. using an empirical force constant model. ${ }^{33}$ They find a maximum $2 \Gamma$ of about $1.5 \mathrm{meV}$ for the longitudinal modes in the [111] direction, which is also in reasonable agreement with our data. In all cases, the linewidths appear to increase with increasing phonon energy.

Examples of phonon DOS from BvK models, damped $\mathrm{BvK}$ models, and reduction of experimental data are shown in Fig. 8. Especially at high temperatures, these DOSs yield slightly different phonon entropies. To leading order in anharmonic perturbation theory, the phonon entropy is given by the quasiharmonic formula [Eq. (19) below] with the shifted energies. ${ }^{41,42}$ This would correspond to using our undamped BvK models. Calculating the entropy using the undamped $\mathrm{BvK}$ models gives a total entropy that is larger than that obtained from reduced experimental data. As the phonon linewidths increase, a particular phonon can be created or annihilated over a wider spectrum of energies. The damping function of Eq. (12) causes an increase in the mean phonon energy and, thus, a decrease in phonon entropy. To minimize data manipulations, we report the entropy from reduced experimental data. We did not correct for the effects of instrument resolution broadening, which causes us to overestimate the phonon entropy by as much as $0.03 k_{B} /$ atom. This is not included in our estimates of the error.

\section{DISCUSSION}

In the quasiharmonic approximation, the phonon entropy is given by 
TABLE III. Longitudinal force constants $\mathcal{L}_{X}$ for the $X \mathrm{NN}$ shell in units of $\mathrm{N} / \mathrm{m}$ as determined by $\mathrm{BvK}$ models and ab initio calculations. Data from Wallis et al. (Ref. 26) and Gilat and Nicklow (Ref. 28) are also tabulated.

\begin{tabular}{|c|c|c|c|c|c|}
\hline $\begin{array}{l}\text { Force } \\
\text { constant }\end{array}$ & $\begin{array}{c}\text { Wallis } \\
\text { Pseudopotential } \\
(0 \mathrm{~K})\end{array}$ & $\begin{array}{c}\text { Empirical } \\
\quad(80 \mathrm{~K})\end{array}$ & $\begin{array}{l}\text { Gilat } \\
(80 \mathrm{~K})\end{array}$ & $\begin{array}{l}\text { esent study } \\
\text { BvK } \\
(10 \mathrm{~K})\end{array}$ & $\begin{array}{l}\text { Plane wave } \\
\quad(0 \mathrm{~K})\end{array}$ \\
\hline $\mathcal{L}_{1}$ & 21.70 & 24.60 & 21.55 & 21.26 & 20.69 \\
\hline $\mathcal{L}_{2}$ & 2.60 & 2.68 & 2.45 & 2.45 & 2.07 \\
\hline $\mathcal{L}_{3}$ & -0.86 & -0.68 & -0.92 & -0.82 & -0.75 \\
\hline
\end{tabular}

$$
S_{\mathrm{ph}}\left(T, T_{p}\right)=3 k_{B} \int_{0}^{\infty} g_{T}\left[\left(n_{T_{p}}+1\right) \ln \left(n_{T_{p}}+1\right)+n_{T_{p}} \ln \left(n_{T_{p}}\right)\right] d E,
$$

where $g_{T}$ is the DOS at temperature $T$ and $n_{T}$ is the mean occupation number for bosons at temperature $T_{p}$

$$
n_{T_{p}}=\frac{1}{\exp (\beta E)-1},
$$

where $\beta=\left(k_{B} T\right)^{-1}$. To calculate the phonon entropy at all temperatures, we find the phonon DOS at temperature $T_{p}$ by linearly interpolating between the two neighboring measurements, and then set $g_{T} \rightarrow g_{T}$ in Eq. (19). To find the harmonic phonon entropy $S_{\mathrm{ph}, \mathrm{H}}$, we use Eq. (19) with $g_{T} \rightarrow g_{10}$ (again, the subscript 10 refers to $10 \mathrm{~K}$ ). The difference between these two quantities is the nonharmonic phonon entropy $S_{\mathrm{ph}, \mathrm{NH}}$. We have

$$
\begin{gathered}
S_{\mathrm{ph}}=S_{\mathrm{ph}}(T)=S_{\mathrm{ph}}(T, T), \\
S_{\mathrm{ph}, \mathrm{H}}=S_{\mathrm{ph}, \mathrm{H}}(T)=S_{\mathrm{ph}}\left(T_{0}, T\right), \\
S_{\mathrm{ph}, \mathrm{NH}}=S_{\mathrm{ph}, \mathrm{NH}}(T)=S_{\mathrm{ph}}(T)-S_{\mathrm{ph}, \mathrm{H}}(T),
\end{gathered}
$$

where we use $T_{0}=10 \mathrm{~K}$. Classically, we may find $S_{\mathrm{ph}, \mathrm{D}}$ using the following equation:

$$
S_{\mathrm{ph}, \mathrm{D}}=S_{\mathrm{ph}, \mathrm{D}}(T)=\int_{T_{0}}^{T} \frac{C_{P}-C_{V}}{T^{\prime}} d T^{\prime}=\int_{T_{0}}^{T} \frac{9 K_{T} \alpha^{2}}{\rho_{N}} d T^{\prime},
$$

where $K_{T}$ is the isothermal bulk modulus, $\alpha$ is the linear coefficient of thermal expansion, and $\rho_{N}$ is the atomic number density, all of which are temperature dependent. Here, we used the temperature dependent isothermal bulk modulus found by $\mathrm{He}$ et al., ${ }^{60}$ and the temperature dependent lattice parameter and linear coefficient of thermal expansion for a "real crystal" found by Wang and Reeber ${ }^{1}$

Using the electronic DOS of Fig. 7, the electronic contribution to the entropy was calculated as

$$
S_{\mathrm{el}}\left(T, T_{p}\right)=-k_{B} \int_{0}^{\infty} \mathcal{D}_{T}\left[f_{T_{p}} \ln \left(f_{T_{p}}\right)+\left(1-f_{T_{p}}\right) \ln \left(1-f_{T_{p}}\right)\right] d E,
$$

where $\mathcal{D}_{T}$ is the electronic DOS at temperature $T$, and $f_{T_{p}}$ is the mean occupation number for fermions at temperature $T_{p}$ :

$$
f_{T_{p}}=\frac{1}{\exp [\beta(E-\mu)]+1} .
$$

At all temperatures, we approximated the chemical potential as the Fermi energy, $\mu=E_{F}$, which should be accurate on the order of $0.01 \% .{ }^{61}$ To get the total electronic entropy, then, we take $\mathcal{D}_{T} \rightarrow \mathcal{D}_{T_{p}}$. The ground state contribution, $S_{\mathrm{el}, \mathrm{G}}$, in analogy with the harmonic contribution of the phonons, is given by taking $\mathcal{D}_{T} \rightarrow \mathcal{D}_{T_{0}}$. The electronic entropy of dilatation $S_{\text {el,D }}$ is given by the difference

$$
\begin{gathered}
S_{\mathrm{el}}=S_{\mathrm{el}}(T)=S_{\mathrm{el}}(T, T), \\
S_{\mathrm{el}, \mathrm{G}}=S_{\mathrm{el}, \mathrm{G}}(T)=S_{\mathrm{el}}\left(T_{0}, T\right), \\
S_{\mathrm{el}, \mathrm{D}}=S_{\mathrm{el}, \mathrm{D}}(T)=S_{\mathrm{el}}-S_{\mathrm{el}, \mathrm{G}},
\end{gathered}
$$

with $T_{0}=0 \mathrm{~K}$.

The electron-phonon entropy was determined from the adiabatic and nonadiabatic electron-phonon free energies calculated by Bock et al. ${ }^{43,44}$ using the thermodynamic relationship

$$
S=-\frac{\partial F}{\partial T}
$$

Forsblom et $a l .{ }^{39}$ have found the entropy and enthalpy of formation of a vacancy in aluminum to be $\Delta S_{\mathrm{vac}, f}=2.35 k_{B}$ and $\Delta H_{\mathrm{vac}, f}=0.75 \mathrm{eV}$, respectively. The configurational entropy $S_{\mathrm{vac}, c}$ and the formation entropy $S_{\mathrm{vac}, f}$ of sets of vacancies are given by

$$
\begin{gathered}
c=c(T)=\exp \left[-\beta\left(\Delta H_{\mathrm{vac}, f}-T \Delta S_{\mathrm{vac}, f}\right)\right] \\
S_{\mathrm{vac}, f}=S_{\mathrm{vac}, f}(T)=c \Delta S_{\mathrm{vac}, f}, \\
S_{\mathrm{vac}, c}=S_{\mathrm{vac}, c}(T)=-k_{B}[c \ln (c)+(1-c) \ln (1-c)],
\end{gathered}
$$

where $c$ is the concentration of vacancies.

All contributions to the entropy are shown in Fig. 9. The total entropy of aluminum was obtained with Eq. (7). Values 


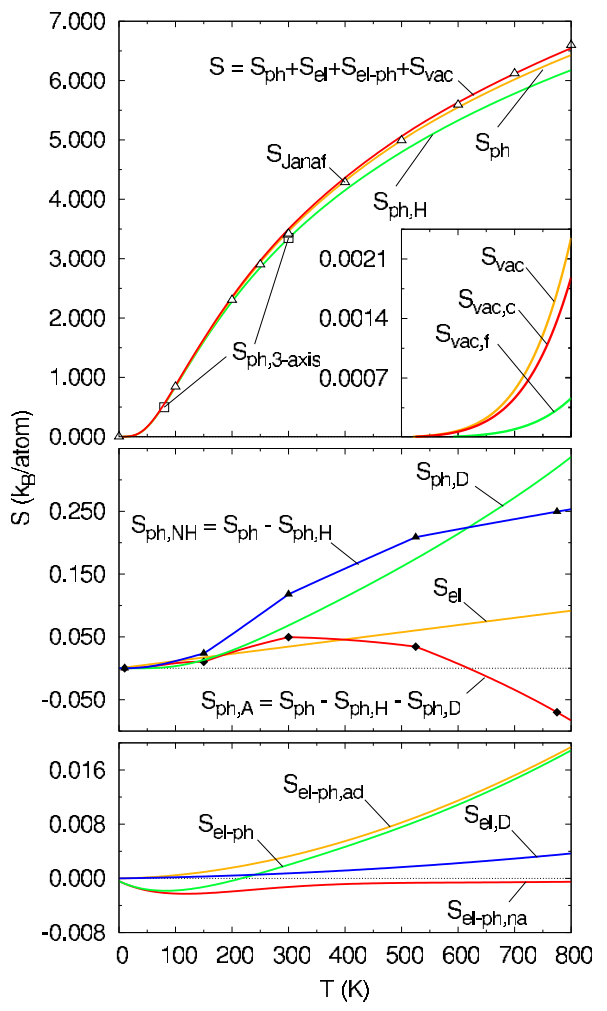

FIG. 9. (Color online) Contributions to the entropy of aluminum as a function of temperature. The temperature labels at the bottom of the plot apply to all panels and the inset. Open triangles are total entropy data taken from the NIST-JANAF thermochemical tables, and open squares are phonon entropy derived from triple-axis data. Closed markers are data from the current experiment; lines are either calculations or interpolations.

for the total entropy were taken from the NIST-JANAF thermochemical tables, ${ }^{62}$ and agreement is excellent, with a rootmean-square deviation of $0.046 k_{B}$ /atom. ${ }^{63}$ We also see excellent agreement between our (time-of-flight based) values for the phonon entropy and those derived using triple-axis data at 80 and $300 \mathrm{~K}$. (The data were taken from Stedman and Nilsson ${ }^{23}$ and used by Gilat and Nicklow ${ }^{28}$ to generate phonon DOS, which we then used to find the phonon entropy.)

The harmonic phonon entropy accounts for most of the entropy of aluminum. The next largest contribution is from the phonon entropy of dilatation, but this is already an order of magnitude smaller. The quasiharmonic model is a useful one for aluminum; nevertheless, the anharmonic phonon entropy is non-negligible and comparable in magnitude to the electronic entropy. The adiabatic electron-phonon interaction is another order of magnitude smaller, followed by the dilatational correction to the electronic entropy. The vacancy contribution to the entropy is primarily configurational and is very small.

Over the temperatures measured, the anharmonic phonon entropy $S_{\mathrm{ph}, A}$ ranges from -0.10 to $+0.08 k_{B}$ /atom (where we have incorporated errors of approximately $\pm 0.03 k_{B} /$ atom). At our highest temperature of $775 \mathrm{~K}$, we have -0.10 $<S_{\mathrm{ph}, A}<-0.04 k_{B}$ /atom. The anharmonic entropy is positive up to around $625 \mathrm{~K}$, after which it becomes negative and decreasing.

The anharmonic contribution to the entropy of aluminum is dominated by phonon-phonon interactions over the much smaller contribution from electron-phonon interactions. ${ }^{31}$ It is interesting that the shape of the anharmonic entropy curve is similar to that reported recently for the fcc nickel, ${ }^{18}$ and the superlinear temperature dependence of $1 / \mathcal{Q}$ is also similar. Aluminum is a simple metal, and nickel is a magnetic $d$-band metal with a complex electronic structure, so it would be surprising if their similar anharmonic behaviors originated from electron-phonon interactions. Phonon-phonon interactions are the probable source of the large increase in phonon linewidth with temperature in both metals. It is interesting that this increase in linewidth is approximately quadratic with temperature, as opposed to the linear effect predicted by the quasiharmonic approximation and perturbation theory. ${ }^{41,64,65}$ The quasiharmonic approximation is expected to be most appropriate when the phonon frequency shifts are small and the phonon lifetimes are long. The latter condition may not apply well to aluminum or nickel.

\section{CONCLUSION}

Measurements of the inelastic scattering of neutrons by phonons in aluminum were made at temperatures of 10,150 , 300,525 , and $775 \mathrm{~K}$. Phonon DOS were obtained from the reduced experimental data, and were used to determine the harmonic, nonharmonic, and total phonon entropy of aluminum. The sum of the phonon, electronic, and vacancy contributions to the entropy agree exceptionally well with accepted values for the total entropy over the entire range of temperatures studied. The anharmonic entropy obtained from the shifts of phonon frequencies was small, but the broadening of the phonon DOS was significant and scaled superlinearly with temperature. The anharmonic behavior was attributed to phonon-phonon interactions. The experimental phonon DOSs were fitted to BvK models of the forces in the solid. A linear decrease with temperature was found for the $1 \mathrm{NN}, 2 \mathrm{NN}$, and $3 \mathrm{NN}$ force constants, with the $1 \mathrm{NN}$ force constants decreasing by approximately $10 \%$ over the range of temperatures measured.

\section{ACKNOWLEDGMENTS}

We thank L. Jirik, K. Littrell, and A. Kolesnikov for their support during our LRMECS beamtime, and L. Daemen for his support during our Pharos beamtime. We also thank R. Stevens for help with experiments, and M. Winterrose and J. McCorquodale for help with the computational effort. This work has benefited from the use of the Intense Pulsed Neutron Source at Argonne National Laboratory and the Los Alamos Neutron Science Center at Los Alamos National Laboratory. These facilities are funded by the U.S. Department of Energy, under Contracts No. W-31-109-Eng-38 and No. DEAC52-06NA25396, respectively. Finally, this work was supported by the Department of Energy through Basic Energy Sciences Grant No. DE-FG02-03ER46055. 
${ }^{1}$ K. Wang and R. R. Reeber, Philos. Mag. A 80, 1629 (2000).

${ }^{2}$ D. C. Wallace, Phys. Rev. 187, 991 (1969).

${ }^{3}$ D. C. Wallace, Phys. Rev. B 1, 3963 (1970).

${ }^{4}$ P. K. Lam and M. L. Cohen, Phys. Rev. B 24, 4224 (1981).

${ }^{5}$ P. K. Lam and M. L. Cohen, Phys. Rev. B 25, 6139 (1982).

${ }^{6}$ P. K. Lam and M. L. Cohen, Phys. Rev. B 27, 5986 (1983).

${ }^{7}$ M. Zoli and V. Bortolani, J. Phys.: Condens. Matter 2, 525 (1990).

${ }^{8}$ G. K. Straub, J. B. Aidun, J. M. Wills, C. R. Sanchez-Castro, and D. C. Wallace, Phys. Rev. B 50, 5055 (1994).

${ }^{9}$ C. Bercegeay and S. Bernard, Phys. Rev. B 72, 214101 (2005).

${ }^{10}$ K. Moriguchi and M. Igarashi, Phys. Rev. B 74, 024111 (2006).

${ }^{11}$ J. Trampenau, W. Petry, and C. Herzig, Phys. Rev. B 47, 3132 (1993).

${ }^{12}$ P. D. Bogdanoff, B. Fultz, J. L. Robertson, and L. Crow, Phys. Rev. B 65, 014303 (2001).

${ }^{13}$ F. Güthoff, B. Hennion, C. Herzig, W. Petry, H. R. Schober, and J. Trampenau, J. Phys.: Condens. Matter 6, 6211 (1994).

${ }^{14}$ W. Petry, A. Heiming, J. Trampenau, M. Alba, C. Herzig, H. R. Schober, and G. Vogl, Phys. Rev. B 43, 10933 (1991).

${ }^{15}$ A. Heiming, W. Petry, J. Trampenau, M. Alba, C. Herzig, H. R. Schober, and G. Vogl, Phys. Rev. B 43, 10948 (1991).

${ }^{16}$ J. Trampenau, A. Heiming, W. Petry, M. Alba, C. Herzig, W. Miekeley, and H. R. Schober, Phys. Rev. B 43, 10963 (1991).

${ }^{17}$ O. Eriksson, J. M. Wills, and D. C. Wallace, Phys. Rev. B 46, 5221 (1992).

${ }^{18}$ M. Kresch, O. Delaire, R. Stevens, J. Y. Y. Lin, and B. Fultz, Phys. Rev. B 75, 104301 (2007).

${ }^{19}$ B. N. Brockhouse and A. T. Stewart, Phys. Rev. 100, 756 (1955).

${ }^{20}$ R. S. Carter, H. Palevsky, and D. J. Hughes, Phys. Rev. 106, 1168 (1957).

${ }^{21}$ B. N. Brockhouse and A. T. Stewart, Rev. Mod. Phys. 30, 236 (1958).

${ }^{22}$ K. E. Larsson, U. Dahlborg, and S. Holmryd, Ark. Fys. 17, 369 (1960).

${ }^{23}$ R. Stedman and G. Nilsson, Phys. Rev. 145, 492 (1966).

${ }^{24}$ C. B. Walker, Phys. Rev. 103, 547 (1956).

${ }^{25}$ G. C. Peterson and T. Smith, J. Phys. F: Met. Phys. 2, 7 (1972).

${ }^{26}$ R. F. Wallis, A. A. Maradudin, V. Bortolani, A. G. Eguiluz, A. A. Quong, A. Franchini, and G. Santoro, Phys. Rev. B 48, 6043 (1993).

${ }^{27}$ R. Stedman, L. Almqvist, and G. Nilsson, Phys. Rev. 162, 549 (1967)

${ }^{28}$ G. Gilat and R. M. Nicklow, Phys. Rev. 143, 487 (1966).

${ }^{29}$ M. Zoli, J. Phys.: Condens. Matter 3, 6249 (1991).

${ }^{30}$ M. I. Katsnel'son, A. V. Trefilov, and K. Yu. Khromov, JETP Lett. 69, 688 (1999).

${ }^{31}$ G. Björkman, I. Lundqvist, and A. Sjölander, Phys. Rev. 159, 551 (1967)

${ }^{32}$ M. Zoli, Philos. Mag. Lett. 62, 203 (1990).

${ }^{33}$ M. Zoli, G. Santoro, V. Bortolani, A. A. Maradudin, and R. F. Wallis, Phys. Rev. B 41, 7507 (1990).

${ }^{34}$ A. Franchini, G. Santoro, V. Bortolani, A. A. Maradudin, and R. F. Wallis, Phys. Rev. B 45, 11982 (1992).

${ }^{35}$ D. A. Ditmars, C. A. Plint, and R. C. Shukla, Int. J. Thermophys. 6, 499 (1985).

${ }^{36}$ R. C. Shukla, C. A. Plint, and D. A. Ditmars, Int. J. Thermophys. 6, 517 (1985).
${ }^{37}$ P. J. Meschter, J. W. Wright, C. R. Brooks, and T. G. Kollie, J. Phys. Chem. Solids 42, 861 (1981).

${ }^{38}$ G. Grimvall, J. Häglund, and A. Fernandéz Guillermet, Phys. Rev. B 47, 15338 (1993).

${ }^{39}$ M. Forsblom, N. Sandberg, and G. Grimvall, Phys. Rev. B 69, 165106 (2004).

${ }^{40}$ D. C. Wallace, Phys. Rev. E 56, 1981 (1997).

${ }^{41}$ D. C. Wallace, Thermodynamics of Crystals (Dover, New York, 1998).

${ }^{42}$ D. C. Wallace, Statistical Physics of Crystals and Liquids: A Guide to Highly Accurate Equations of State (World Scientific, Singapore, 2002).

${ }^{43}$ N. Bock, D. Coffey, and D. C. Wallace, Phys. Rev. B 72, 155120 (2005).

${ }^{44}$ N. Bock, D. C. Wallace, and D. Coffey, Phys. Rev. B 73, 075114 (2006).

${ }^{45}$ P. B. Allen and J. C. K. Hui, Z. Phys. B 37, 33 (1980).

${ }^{46}$ M. Thiessen, Int. J. Thermophys. 7, 1183 (1986).

${ }^{47}$ B. T. Fultz and J. M. Howe, Transmission Electron Microscopy and Diffractometry of Materials, 2nd ed. (Springer-Verlag, Berlin, 2002), Chap. 1, p. 51.

${ }^{48} \mathrm{~S}$. W. Lovesey, Theory of Neutron Scattering from Condensed Matter (Clarendon, Oxford, 1984), Vol. 1, p. 301.

${ }^{49}$ The transform is the same as a convolution, except that the width of the broadening function is energy dependent. We assumed that $R * D \approx D * R$, which is reliable for our conditions of use.

${ }^{50} \mathrm{M}$. Born and K. Huang, Dynamical Theory of Crystal Lattices (Clarendon, Oxford, 1988).

${ }^{51}$ A. Maradudin, E. Montroll, G. Weiss, and I. Ipatova, in Solid State Physics, 2nd ed., edited by H. Ehrenreich, F. Seitz, and D. Turnbull (Academic, New York, 1971), Suppl. 3.

${ }^{52} \mathrm{G}$. Venkataraman, L. A. Feldkamp, and V. C. Sahni, Dynamics of Perfect Crystals (MIT, Cambridge, 1975).

${ }^{53}$ A. F. Yue, I. Halevy, A. Papandrew, P. D. Bogdanoff, B. Fultz, W. Sturhahn, E. E. Alp, and T. S. Toellner, Hyperfine Interact. 141, 249 (2002).

${ }^{54}$ G. Kresse and J. Furthmüller, Comput. Mater. Sci. 6, 15 (1996).

${ }^{55}$ G. Kresse and D. Joubert, Phys. Rev. B 59, 1758 (1999).

${ }^{56}$ J. P. Perdew, K. Burke, and M. Ernzerhof, Phys. Rev. Lett. 77, 3865 (1996).

${ }^{57}$ H. J. Monckhorst and J. D. Pack, Phys. Rev. B 13, 5188 (1976).

${ }^{58}$ D. Alfè, http://chianti.geol.ucl.ac.uk/ dario

${ }^{59}$ Here, we have used the formula from Ref. 22 with $\left\langle E_{300}\right\rangle$ from our experiment.

${ }^{60}$ D. He, Y. Zhao, L. L. Daemen, J. Qian, K. Lokshin, T. D. Shen, J. Zhang, and A. C. Lawson, J. Appl. Phys. 95, 4645 (2004).

${ }^{61}$ N. W. Ashcroft and N. D. Mermin, Solid State Physics (Thomas Learning, Springfield, IL, 1976), Chap. 2, pp. 46-47.

${ }^{62}$ M. W. Chase, J. Phys. Chem. Ref. Data Monogr. 9, 59 (1998).

${ }^{63}$ The root-mean-square deviations for the entropy as determined from the BvK models and from the damped BvK models were 0.066 and $0.039 k_{B}$ /atom, respectively. The differences between these three models are small; nevertheless, it may be that using a damped DOS (without resolution broadening) more accurately represents the phase space covered by the anharmonic oscillators.

${ }^{64}$ A. A. Maradudin and A. E. Fein, Phys. Rev. 128, 2589 (1962).

${ }^{65}$ R. A. Cowley, Adv. Phys. 13, 421 (1963). 